\title{
Recent results from the ALICE Experiment at LHC
}

\author{
Catalin Ristea $^{1, *}$ for the ALICE Collaboration \\ ${ }^{1}$ Institute of Space Science, Bucharest-Magurele RO-077125, Romania
}

\begin{abstract}
ALICE (A Large Ion Collider Experiment) at the LHC performed high statistics measurements in $\mathrm{Pb}-\mathrm{Pb}$ collisions at the top LHC energy, complemented with large recent reference datasets in elementary proton-proton collisions at the same energy. Elementary pp collisions are serving as baseline for testing QCD properties and allow the study of the changes induced by the hot and dense medium produced in heavy ion collisions. Key observables like nuclear modification factors, jet production, flow phenomena and spectra for identified particles, related to the different stages of collision evolution, are presented and compared with the most recent results from $\mathrm{p}-\mathrm{Pb}$ and $\mathrm{Xe}-\mathrm{Xe}$ collisions, thus allowing to probe both initial cold nuclear matter and final state effects, combined with the system size dependence of the measurements.
\end{abstract}

\section{Introduction}

Nuclear collisions at relativistic energies produce a strongly interacting matter under extreme conditions of temperature and energy density. In these collisions, a deconfined state of quarks and gluons (Quark Gluon Plasma - QGP)[1,2] is expected to be formed.

ALICE (A Large Ion Collider Experiment) is one of the four large experiments at the LHC (Large Hadron Collider) and is dedicated to the study of heavy-ion collisions. The ALICE detector system and performance are described in detail in [3]. The central barrel tracking detectors (ITS - Inner Tracking System and TPC - Time Projection Chamber) are located inside a soleinoid magnet, which provide an uniform magnetic field of $0.5 \mathrm{~T}$ parallel to the beam axis for momentum measurements. Two V0 scintillator counters, covering 2.8 $<\eta<5.1$ and $-3.7<\eta<-1.7$, provide information for trigering, event selection and collision centrality determination. ALICE detector provide excellent particle identification (PID) for hadrons, leptons and photons over a wide momentum range. Hadron PID is made using TPC, ITS, TOF (Time of Flight detector) and HMPID (High Momentum Particle Identification Detector) detectors. Electron identification is achieved in a Transition Radiation Detector (TRD), while the high momentum neutral particles at midrapidity are identified with ElectroMagnetic Calorimeter (EMCal).

\section{Bulk Particle Production}

The charged particle multiplicity is a key observable to characterize the properties of the matter created in relativistic heavy-ion collisions. The dependence of pseudorapidity density

*e-mail: catalin.ristea@cern.ch 
of charged particles, $\left\langle d N_{c h} / d \eta\right\rangle$, on colliding system, energy and centrality can provide information about relative contributions to particle production from hard scatterings and soft processes and about the energy density achieved in these collisions.

The mid-rapidity charged-particle multiplicity normalised by the number of nucleon pairs participating in the collision as a function of the number of participants is presented in the left part of Figure 1 [4]. The data are from pp, p- $\mathrm{Pb}, \mathrm{Xe}-\mathrm{Xe}, \mathrm{Pb}-\mathrm{Pb}$ collisions at LHC energies $\left(\sqrt{s_{N N}}=2.76\right.$ and $\left.5.02 \mathrm{TeV}\right)$ and $\mathrm{Cu}-\mathrm{Cu}, \mathrm{Au}-\mathrm{Au}$ collisions at RHIC top energy $\left(\sqrt{s_{N N}}=\right.$ $0.2 \mathrm{TeV}$ ). The average multiplicity densities scaled by the number of participant pairs show a slight increase with $N_{\text {part }}$. For more central collisions, Xe-Xe results are higher than $\mathrm{Pb}-\mathrm{Pb}$ results at similar number of participants. The same behavior was observed also at RHIC, where $\mathrm{Cu}-\mathrm{Cu}$ multiplicities are larger than in $\mathrm{Au}-\mathrm{Au}$ for most central collisions [5].
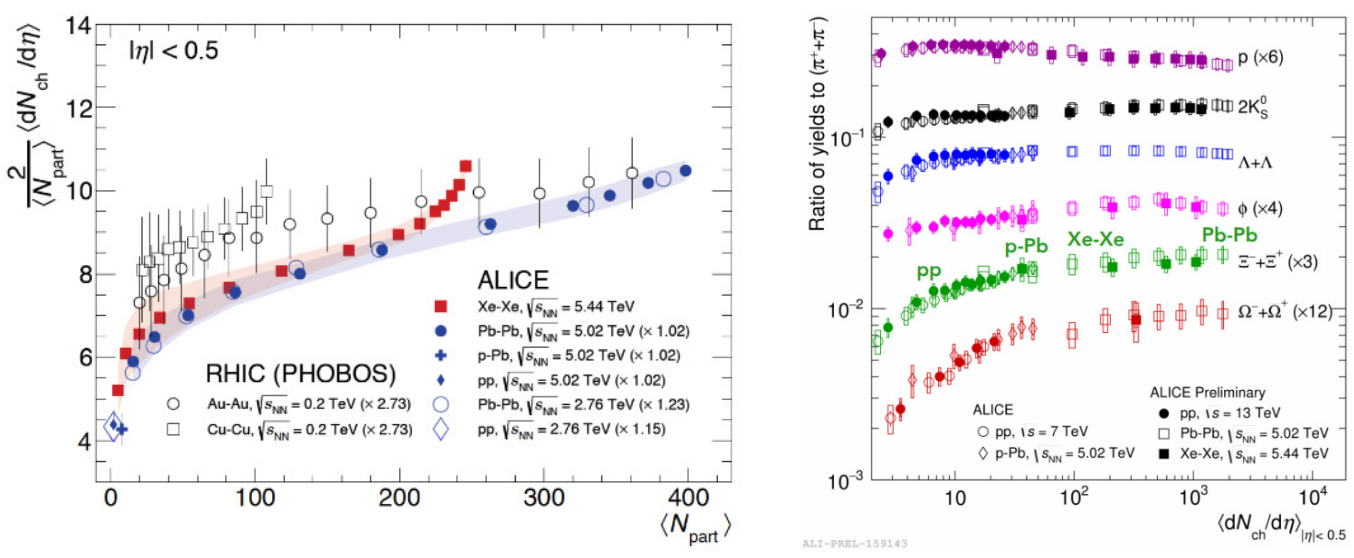

Figure 1. Left: The $\frac{2}{\left\langle N_{\text {part }}\right\rangle}\left\langle d N_{c h} / d \eta\right\rangle$ as a function of number of participants. Figure is taken from [4]. Right: $p_{T}$-integrated yield ratios to pions as a function of charged particle multiplicity density measured in $|y|<0.5$ [7].

Strangeness enhancement was originally proposed as a signature of QGP formation [6] and has been observed in heavy-ion collisions at relativistic energies [16]. Figure 1 (right panel) presents the ratios of the yields of $K_{S}^{0}, \Lambda, \phi, \Xi$ and $\Omega$ to charged pion yield $\left(\pi^{+}+\pi^{-}\right)$as a function of $\left\langle d N_{c h} / d \eta\right\rangle$ [7]. Data from pp and p-Pb collisions show an enhancement of strange and multi-strange compared to non-strange particle production, while in AA collisions no significant change with multiplicity was observed. For similar multiplicity, the results do not show any dependence of the system or the collision energy.

The kinetic freeze-out parameters (the kinetic freeze-out temperature, $T_{k i n}$ and the transverse collective flow velocity, $\beta_{T}$ ) are obtained by blast-wave fits to $p_{T}$ spectra. The blastwave model[8] assumes that the system is in local thermal equilibrium and the produced particles are moving with a common collective transverse flow velocity and freeze-out (stop interacting) at a temperature $T_{k i n}$. The 1- $\sigma$ contours in the $T_{k i n}-\beta_{T}$ plane, as well as the blastwave fit parameters, $T_{k i n}$ and $\beta_{T}$, are presented in Figure 2 (left panel). In AA collisions, $\beta_{T}$ increases from peripheral to central collisions, indicating a stronger expansion in more central collisions. In $\mathrm{pp}$ and $\mathrm{p}-\mathrm{Pb}$ we observe similar evolution towards high multiplicity. The freeze-out temperature decreases in more central collisions suggesting that the fireball expands and decouples later in time. 

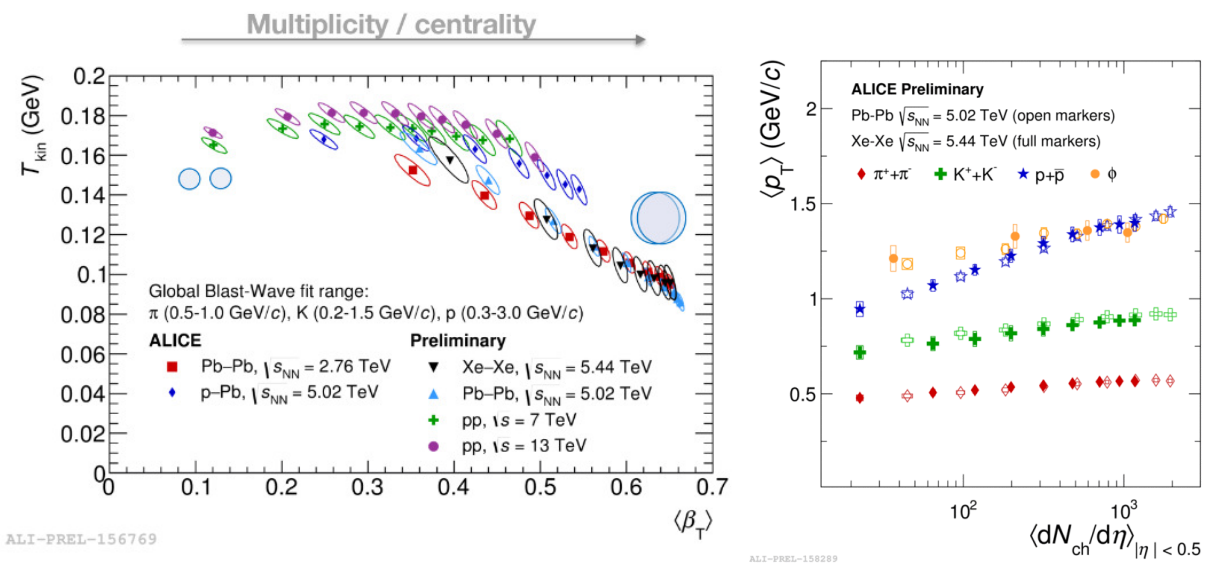

Figure 2. Left: Contours in $T_{k i n}-\beta_{T}$ plane showing the 1- $\sigma$ deviation lines from the minimum $\chi^{2}$ values, as well as the blast-wave fit parameters, $T_{k i n}$ and $\beta_{T}$ for various collision systems and energies. Right: Centrality dependence of $\left\langle p_{T}\right\rangle$ for pions, kaons, protons and $\phi$ mesons in $\mathrm{Pb}-\mathrm{Pb}$ and $\mathrm{Xe}-\mathrm{Xe}$ collisions.

Another observable sensitive to the collective expansion is the average transverse momentum $\left\langle p_{T}\right\rangle$ of the emitted particles. It is found that $\left\langle p_{T}\right\rangle$ for all studied particle species increases from peripheral to most central collisions. For both $\mathrm{Pb}-\mathrm{Pb}$ and $\mathrm{Xe}-\mathrm{Xe}$ collisions, an increase in $\left\langle p_{T}\right\rangle$ with the particle mass is observed and this dependence reflects the radial collective expansion of the produced systems (Figure 2). The difference in $\left\langle p_{T}\right\rangle$ values between $p$ and $\pi / K$ is larger for higher multiplicities suggesting that the average radial collective flow is stronger in more central collisions. For the $\phi$ meson that has similar mass as the proton, the behavior is the same in central collisions.

\section{Anisotropic flow}

In a non-central nuclear collision, the overlap region between the two colliding nuclei is not spherically symmetric. The almond shaped interaction volume produce an asymmetric pressure gradients, which converts the initial-state geometric anisotropy to a final state momentum-space anisotropy. Anisotropic flow is characterized by the coefficients, $v_{n}$, in the Fourier expansion of the particle azimuthal distribution. The second harmonic coefficient, $v_{2}$, is called elliptic flow.

The $v_{2}\left(p_{T}\right)$ of $\pi^{ \pm}, K^{ \pm}, p+\bar{p}, \phi$-meson, $K_{S}^{0}$ and $\Lambda+\bar{\Lambda}$ produced in $\mathrm{Pb}-\mathrm{Pb}$ collisions at $\sqrt{s_{N N}}=5.02 \mathrm{TeV}$ for various centrality classes are shown in Figure 3. All the $v_{2}$ values increase from central to peripheral collisions up to the $40-50 \%$ centrality interval for all particle species. In the low- $p_{T}$ region $\left(p_{T}<3 \mathrm{GeV} / \mathrm{c}\right)$, the elliptic flow coefficient of identified particles exhibits mass-ordering behavior due to the interplay between radial and anisotropic flow (at the same $p_{T}$, heavier species have a lower $v_{2}$ than lighter ones). In the intermediate $p_{T}$ range $\left(3<p_{T}<8-10 \mathrm{GeV} / \mathrm{c}\right)$, particles group according to their type, mesons or baryons, indicating quark coalescence as a possible hadron production mechanism in this region. At high $p_{T}\left(p_{T}>10 \mathrm{GeV} / \mathrm{c}\right)$, the magnitude of $v_{2}$ is similar for pions and protons within uncertainties.

To test particle type scaling and mass ordering, the $\phi$-meson has an important role as its mass is close to the proton mass. In a collective hydrodynamic expansion of the system, 
the $p_{T}$ spectra of particles are determined by thermal freeze-out temperature, flow velocity and particle mass, therefore we expect similar spectra for proton and $\phi$-meson due to similar masses. At low $p_{T}$, the $v_{2}$ of the $\phi$-meson follows the $p+\bar{p} v_{2}$, while at intermediate $p_{T}$ follows the $\pi^{ \pm} v_{2}$.

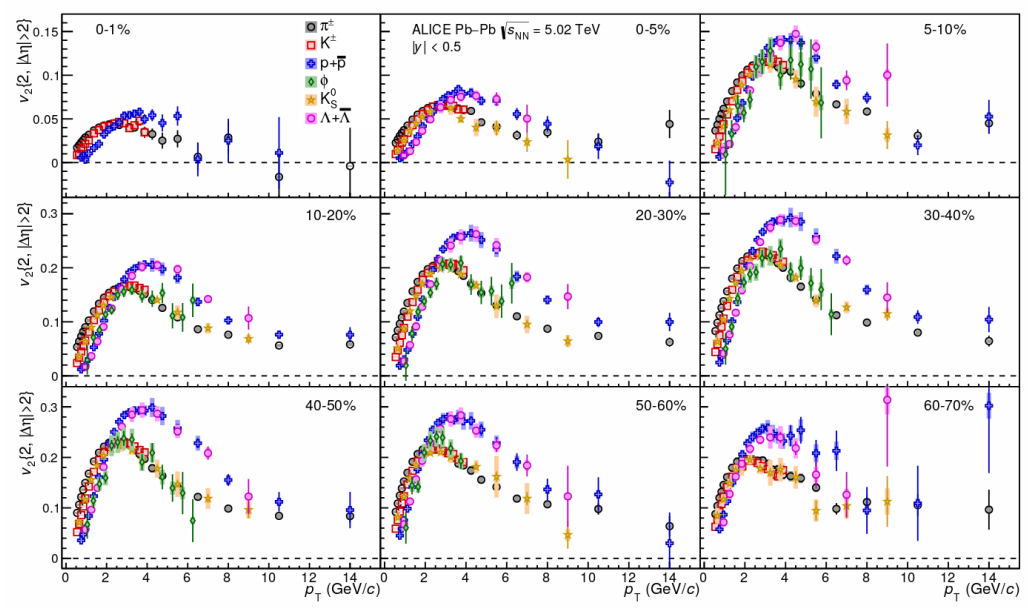

Figure 3. The elliptic flow coefficient of $\pi^{ \pm}, K^{ \pm}, p+\bar{p}, \phi$-meson, $K_{S}^{0}$ and $\Lambda+\bar{\Lambda}$ as a function of transverse momentum for different centrality classes. Statistical and systematic uncertainties are shown as bars and boxes, respectively. Figure is taken from [9].

\section{Hard probes}

\subsection{High-pT suppresion}

Energetic high- $p_{T}$ partons originating from the hard scatterings of the incoming partons propagate through the hot and dense medium produced in the collision and lose energy via collisional and radiative processes. The magnitude of energy loss is sensitive to the medium energy density and the parton path length.

An observable for quantifying medium effects in heavy-ion collsions is the nuclear modification factor, $R_{A A}$ :

$$
R_{A A}=\frac{d^{2} N / d p_{T} d \eta}{T_{A A} d^{2} \sigma^{p p} / d p_{T} d \eta}
$$

where $d^{2} N / d p_{T} d \eta$ is the differential yield per event in the A+A collision, $T_{A A}=\left\langle N_{b i n}\right\rangle / \sigma_{\text {inel }}^{p p}$ is the average nuclear overlap function which describes the nuclear geometry, $\left\langle N_{b i n}\right\rangle$ is the average number of binary nucleon-nucleon collisions, $\sigma_{\text {inel }}^{p p}$ is the inelastic nucleon-nucleon inelastic cross-section and $d^{2} \sigma^{p p} / d p_{T} d \eta$ is the production cross section in pp collisions at the same energy. In the absence of nuclear effects, hard processes are expected to scale with the number of binary collisions and $R_{A A}$ equals unity. If $R_{A A}<1$, the AA yields at a given $p_{T}$ is suppressed compared with expected value from the scaled pp yields.

Comparison of nuclear modification factors in Xe-Xe collisions at $\sqrt{s_{N N}}=5.44 \mathrm{GeV}$ and $\mathrm{Pb}-\mathrm{Pb}$ collisions at $\sqrt{s_{N N}}=5.02 \mathrm{GeV}$ is presented in Figure 4. In the left panel, the $R_{A A}\left(p_{T}\right)$ comparison is made at similar charged particle multiplicity density (similar medium 

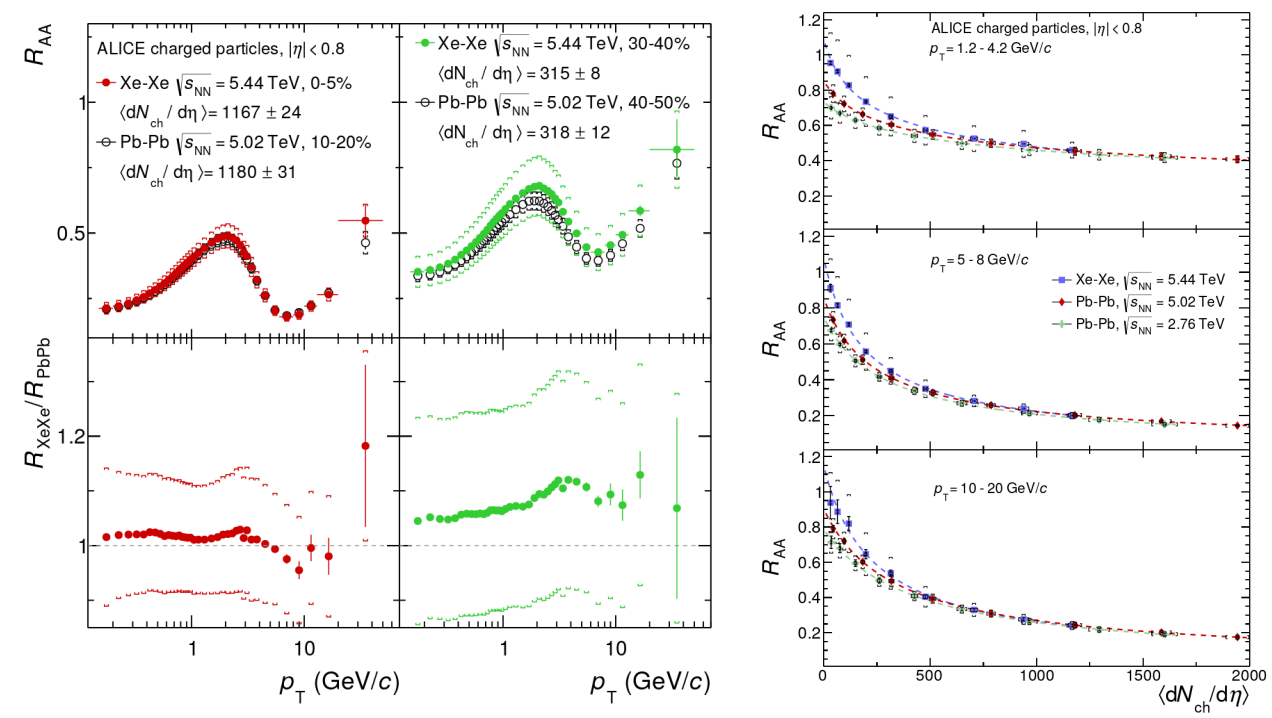

Figure 4. Left: $R_{A A}$ in Xe-Xe collisions (filled circles) and $\mathrm{Pb}-\mathrm{Pb}$ col- lisions (open circles) for similar ranges in $\left\langle d N_{c h} / d \eta\right\rangle$ for the $0-5 \%$ and $30-40 \% \mathrm{Xe}-\mathrm{Xe}$ centrality classes. The vertical lines (brackets) represent the statistical (systematic) uncertainties [10]. Right: Nuclear modification factor in Xe-Xe and $\mathrm{Pb}-\mathrm{Pb}$ collisions integrated over identical regions in $\mathrm{pT}$ as a function of $\left\langle d N_{c h} / d \eta\right\rangle$ [10].

density). For most central Xe-Xe collisions ( $0-5 \%$ centrality), the nuclear modification factor is comparable with $R_{A A}$ obtained in $10-20 \% \mathrm{~Pb}-\mathrm{Pb}$ collisions. For the semi-central collisions (30-40\% Xe-Xe vs. $40-50 \% \mathrm{~Pb}-\mathrm{Pb}$ ), the results are in agreement within uncertainties. In the right panel, the $R_{A A}$ dependence on average charged-particle multiplicity density is compared for three $p_{T}$ intervals (low, medium and high). Again, a similarity in $R_{A A}$ is seen at the same $\left\langle d N_{c h} / d \eta\right\rangle$ for more central collisions $\left(\left\langle d N_{c h} / d \eta\right\rangle>400\right)$. These results could provide new input to constrain path length dependence of the energy loss.

Heavy quarks (charm and beauty), because of their large mass, are produced mainly in the early stages of nuclear collisions and therefore experience the entire evolution of the created medium. As all other energetic partons, the $\mathrm{c}$ and $\mathrm{b}$ quarks interact with the medium constituents via collisional (elastic) and radiative (inelastic) processes. The radiative energy loss is smaller for heavy than for light quarks due to the so-called dead-cone effect[11].

Quarkonia are bound states of heavy quark-antiquark pairs (charmonia, cē and bottomonia, $b \bar{b}$ ). The color potential in quarkonia is screened in the QGP leading to a suppression of their production. The screening depends on the radius of the quarkonium state and therefore on its binding energy, which results in a prediction of sequential suppression with increasing medium temperature $[13,14]$. At the LHC energies, there is an increased contribution due to recombination processes from the larger charm quark density, leading to a smaller suppression. In the case of open heavy flavour, the presence of the medium creates a competition between fragmentation (one quark producing a spray of hadrons - jet) and recombination (the $\mathrm{c}$ quark picks up a light antiquark from the medium).

The $p_{T}$-differential $R_{A A}$ of $\mathrm{D}_{s}^{+}$and average $R_{A A}$ of prompt $\mathrm{D}^{0}, \mathrm{D}^{+}, \mathrm{D}^{*+}$ measured in central $0-10 \% \mathrm{~Pb}-\mathrm{Pb}$ collisions at $\sqrt{s_{N N}}=5.02 \mathrm{TeV}$ [12] is shown in the left panel of Figure 5 . The average non-strange D-meson $R_{A A}$ shows a suppression in most central events up to a factor about 5 for $p_{T}>5 \mathrm{GeV} / \mathrm{c}$. The prompt $\mathrm{D}_{S}^{+} R_{A A}$ is larger, although compatible within 

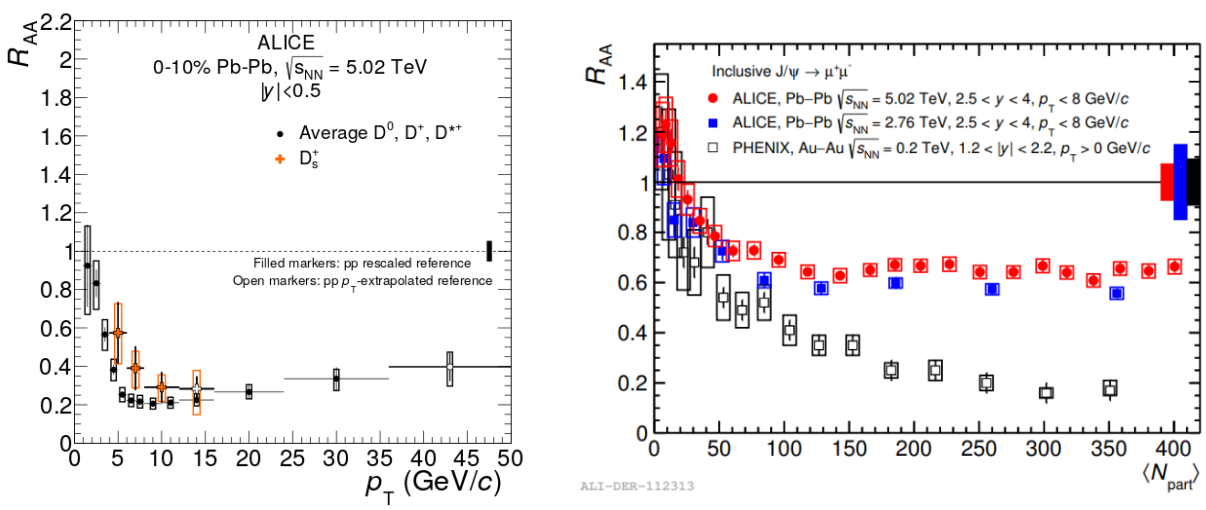

Figure 5. Left: Average $R_{A A}$ of prompt $\mathrm{D}^{0}, \mathrm{D}^{+}, \mathrm{D}^{*+}$ mesons and $R_{A A}$ of $\mathrm{D}_{s}^{+}$in $0-10 \% \mathrm{~Pb}-\mathrm{Pb}$ collisions at $5.02 \mathrm{TeV}$ [12]. Right: Centrality dependence of $J / \Psi$ nuclear modification factor in $\mathrm{Au}-\mathrm{Au}$ and $\mathrm{Pb}-\mathrm{Pb}$ collisions.

uncertainties, than non-strange D-meson $R_{A A}$ in the full measured $p_{T}$ range. These results provide a hint of charm hadronisation via coalescence (the recombination of the c quark in the QGP where s quarks are abundant).

In the right panel of Figure 5, $J / \Psi$ nuclear modification factor in $\mathrm{Au}-\mathrm{Au}$ and $\mathrm{Pb}-\mathrm{Pb}$ collisions as a function of collision centrality is presented . At RHIC top energy, the $J / \Psi$ production is strongly suppressed and the suppression increases with the number of participants. At LHC energies, the data show a smaller suppression for central collisions compared to RHIC results and a weaker centrality dependence. This reduced suppression at LHC energies is an evidence for increasing recombination in $\mathrm{Pb}-\mathrm{Pb}$ collisions. Furthermore, the $R_{A A}$ has constant value over a broad range in centrality and seems both recombination and suppression are decreasing similarly with decreasing centrality.

\subsection{Jets}

High transverse momentum partonic interactions in early stages of nuclear collisions lead to a production of two high- $p_{T}$ back-to-back partons which subsequently evolve as parton showers, hadronize, and are experimentally observed as jets events in the detector. Jets play an important role in studying QGP properties produced in these collisions because during their propagation through the hot and dense medium, the interaction with the colored medium will lead to parton energy loss (jet quenching) [15].

Figure 6 shows the jet nuclear modification factor, defined as the ratio of the measured jet spectra in $\mathrm{Pb}-\mathrm{Pb}$ collisions at $\sqrt{s_{N N}}=5.02 \mathrm{TeV}$ to the pp reference scaled by the number of binary collisions. The measured jet $R_{A A}$ is significantly smaller than unity, indicating a strong suppression of jets in most central $0-10 \% \mathrm{~Pb}-\mathrm{Pb}$ collisions. The suppression is strongest at lower jet transverse momentum, while at high $p_{T}$ the $R_{A A}$ increases slowly. With increasing $p_{T}$, more partons loose more energy and build-up a constant $R_{A A}$.

\section{Summary/Conclusions}

ALICE provides a plethora of precision measurements for many hadron types allowing better understanding and validation of QCD for LHC collisions. Data showed a strangeness enhancement in smaller systems (pp and $\mathrm{pA}$ collisions). The largest radial flow velocities in 


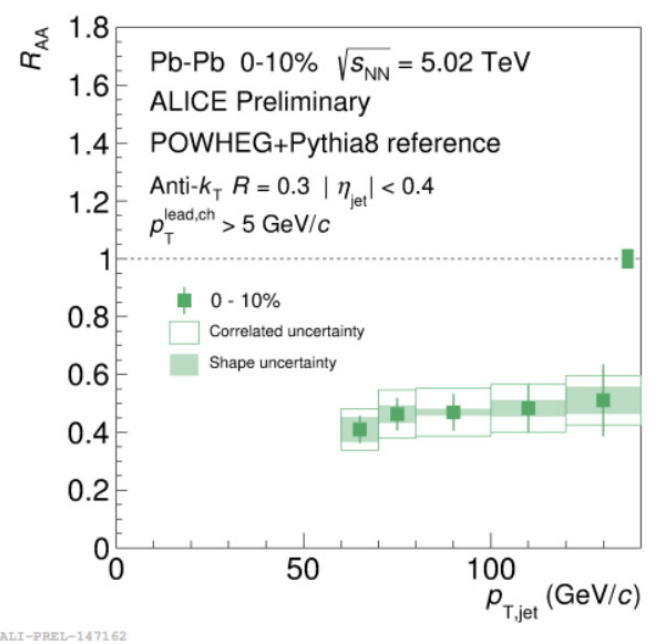

Figure 6. Nuclear modification factor of jets as a function of the jet $p_{T}$ in $0-10 \% \mathrm{~Pb}-\mathrm{Pb}$ collisions at $5.02 \mathrm{TeV}$.

$\mathrm{Pb}-\mathrm{Pb}$ collisions, while $\mathrm{Xe}-\mathrm{Xe}$ results are consistent with $\mathrm{Pb}-\mathrm{Pb} . J / \Psi$ nuclear modification factor at LHC energies showed a smaller suppression than the RHIC results suggesting the increase of recombination processes due to abundance of $\mathrm{c}$ and $\overline{\mathrm{c}}$ quarks at these energies. In most central collisions, the $R_{A A}$ of jets are strongly suppressed due to interaction with the hot and dense medium, therefore these results will place stronger constrains on quark-medium interactions.

\section{References}

[1] E. V. Shuryak, Phys. Rept. 61, 71 (1980)

[2] J. C. Collins and M. J. Perry, Phys. Rev. Lett. 34, 1353 (1975).

[3] ALICE Collaboration, K. Aamodt, JINST 3, S08002 (2008)

[4] ALICE Collaboration, S. Acharya et al., arXiv: 1805.04432 [nucl-ex]

[5] PHOBOS Collaboration, B. Alver et al., Phys. Rev. C 83, 024913 (2011), arXiv: 1011.1940 [nucl-ex]

[6] P. Koch, B. Muller, and J. Rafelski, Phys. Rept. 142, 167 (1986)

[7] ALICE Collaboration, J. Adam et al., Nature Physics 13, 535 (2017), arXiv: 1606.07424 [nucl-ex]

[8] E. Schnedermann et al., Phys. Rev. C 48, 246 (1993)

[9] ALICE Collaboration, S. Acharya et al., arXiv: 1805.04390 [nucl-ex]

[10] ALICE Collaboration, S. Acharya et al., arXiv: 1805.04399 [nucl-ex]

[11] Y. L. Dokshitzer and D. Kharzeev, Phys. Lett. B 519, 199 (2001).

[12] ALICE Collaboration, S. Acharya et al., arXiv: 1804.09083 [nucl-ex]

[13] T. Matsui and H. Satz, Phys. Lett. B 178, 416 (1986)

[14] S. Digal, P. Petreczky, and H. Satz, Phys. Rev. D 64, 094015 (2001), arXiv:hepph/0106017 [hep-ph] 
[15] M. Gyulassy and M. Plumer, Phys. Lett. B 243, 432 (1990); X.-N. Wang and M. Gyulassy, Phys. Rev. Lett. 68, 1480 (1992)

[16] NA57 Collaboration, F. Antinori et al., Phys. Lett. B595, 68 (2004), arXiv:nuclex/0403022 [nucl-ex].

[17] STAR Collaboration, B. I. Abelev et al., Phys. Rev. C77, 04490 (2008), arXiv:0705.2511 [nucl-ex]. 